\title{
Multiple Infantile Myofibromatosis with Skeletal Abnormalities
}

\author{
Ibrahim Abdulaziz Hamama1,2,3*, Tariq Alhazmi'2,3, Waseemoddin Patel ${ }^{1}$ \\ ${ }^{1}$ Division of Neonatology, Department of Pediatrics, King Abdullah Bin Abdulaziz University Hospital, Princess Nora Bint \\ Abdulrahman University, Riyadh, Saudi Arabia \\ ${ }^{2}$ Department of Pediatrics, AlNoor Specialist Hospital, Mecca, Saudi Arabia \\ ${ }^{3}$ Department of Pediatrics, Maternity and Children Hospital, Mecca, Saudi Arabia \\ Email: *iahamama@kaauh.edu.sa
}

How to cite this paper: Hamama, I.A., Alhazmi, T. and Patel, W. (2020) Multiple Infantile Myofibromatosis with Skeletal Abnormalities. Health, 12, 849-856. https://doi.org/10.4236/health.2020.127062

Received: May 29, 2020

Accepted: July 24, 2020

Published: July 27, 2020

Copyright () 2020 by author(s) and Scientific Research Publishing Inc. This work is licensed under the Creative Commons Attribution International License (CC BY 4.0).

http://creativecommons.org/licenses/by/4.0/

(c) (i) Open Access

\begin{abstract}
Background: Infantile myofibromatosis is an uncommon disorder characterized by multiple fibromatous tumours involving skin, bone, muscle, viscera and subcutaneous tissue. It is a rare benign mesenchymal tumour; most commonly occurs in infancy or early childhood. The clinical presentation can mimic malignant tumours of infancy or childhood. Case Presentation: We describe a rare case of multicentric Infantile myofibromatosis in a 6-monthold infant presenting with multiple scalp swellings and associated skeletal abnormalities (adducted thumbs, clinodactyly and bilateral hallux valgus deformity of great toes). The case required surgical excision of all scalp lesions and orthopedic manipulation of skeletal abnormalities. Conclusion: Infantile myofibromatosis presenting as multiple lesions in the scalp associated with skeletal abnormalities, is very rare. To best of our knowledge, the unique combination of the distinct skeletal abnormalities in infantile myofibromatosis has not been reported so far. This report emphasizes the possibility of skeletal abnormalities in infantile myofibromatosis.
\end{abstract}

\section{Keywords}

Infantile Myofibromatosis, Multiple Fibromatous, Skeletal Abnormalities, Clinodactyly, Hallux Valgus Deformity

\section{Introduction}

Infantile myofibromatosis is a rare disorder with multiple fibromatosis tumours in skin bone, muscle, viscera, and subcutaneous tissue [1]. It usually presents in infancy or early childhood. Although rare, it is the most frequent fibrous tumour of infancy [1]. The clinical presentation can mimic malignant tumours of infancy or childhood. Infantile myofibromatosis usually presents as firm, purple or 
flesh-colored nodules in the skin and subcutaneous tissue. The lesions can be solitary or multicentric. Most cases are limited to the skin, but visceral involvement is noted in many instances [2]. The most commonly affected body areas are the head, the neck, and the trunk. The exact etiology of the condition is unknown,

Imaging studies are required to visualize the extent of the growth, the progression of the growth, and to diagnose recurrence of growth, and to aid surgical procedures. A definitive diagnosis of infantile myofibromatosis requires removal and microscopic examination of affected tissue to determine the pathology and to distinguish from the disorders which can mimic like infantile myofibromatosis [3]. Molecular genetic testing can detect mutations in specific genes known to cause the disease. Surgical excision of the lesion in treatment of choice for solitary lesions, treatment for multicentric lesion is not well defined [3].

In this case report, we describe the clinical presentation, radiographic findings, histological examination of an infant with Infantile myofibromatosis and rare associated distinct skeletal abnormalities.

\section{Case Report}

A 6-month-old infant girl presented with multiple swelling in the scalp noted at one month of age. The swelling involved multiple areas of scalp region, temporal and occipital regions, five in number, size varying from $2 \mathrm{~cm}$ to $5 \mathrm{~cm}$. The onset was insidious, and the progression was gradual. A history of spontaneous resolution of two swellings also noted. There was no history of trauma, fever, vomiting, weight loss, feeding concerns, breathing abnormalities, jaundice, cyanosis or pallor.

On examination, the vital signs were stable. A total of five swellings noted in the scalp region. Two were big, $4 \times 5 \mathrm{~cm}$ in dimension, and three were small around $2 \times 3 \mathrm{~cm}$ in size. The swellings were hard and immobile. The skin over the swelling was normal. No significant lymphadenopathy noted. Apart from these lesions, the infant had some atypical skeletal features; the thumbs were in an adducted position, she had bilateral hallux valgus abnormality of the great toes and bilateral clinodactyly (Figure 1). No other dysmorphic features were seen.

A complete blood count showed a hemoglobin of $9.1 \mathrm{~g} / \mathrm{L}$. The rest of the CBC was unremarkable. Blood chemistry was within normal limits. An X-ray of hand and feet revealed; X-Ray (both hands): AP view of both hands showing bilateral in-drawing of the thumbs, in keeping with the appearance of the thumb adduction seen on the clinical examination of the hands, with no other bony or soft tissue lesion. X-Ray (both feet): AP view of both feet showing bilateral hallux valgus deformity in keeping with the appearance of the big toe adduction seen on the clinical examination of the feet. Otherwise, no bony lesions or soft tissue nodules (Figure 1).

A CT-scan showed; CT brain: Axial views showing multiple subcutaneous soft tissue masses involving the skull vault at the level of the left Parietal and occipital 
region. Another relatively larger soft tissue mass is seen in the midline at the level of the posterior fossa. These lesions showed no associated bony changes. Moreover, no intracranial abnormality was detected. An MRI head showed; MRI Brain: T1 weighted sequences Axial and Coronal views showing a mass within the soft tissues of the left parietal region with intact outer table of the skull vault (coronal view) and another relatively larger one in the midline at the level of craniocervical junction region, with no involvement of the underlying soft tissues or spinal canal (Figure 2).

The Neurosurgical team saw the infant after the imaging, and all the five swellings were excised. A histopathologic examination revealed that each nodule consisted of a central and peripheral area, the peripheral area consists of spindle cells (myofibroblasts) with eosinophilic cytoplasm and ovoid nuclei arranged in well-demarcated short bundles and fascicles resembling smooth muscle. The central portion consists of less differentiated rounder cells with pale cytoplasm and basophilic, small round nuclei (Figure 3). The features were consistent with a diagnosis of Infantile myofibromatosis.

Post-surgical excision of the scalp lesions, we refer the infant to the orthopedic team for the abnormal skeletal features. They started the conservative treatment for the adducted thumbs (manual stretching) and advised the parents to follow-up later for definitive surgical correction of the adducted thumb and hallux valgus abnormality of the great toes. Nevertheless, when enquired recently, the patient did not return for follow-up, and we lost track of the patient.

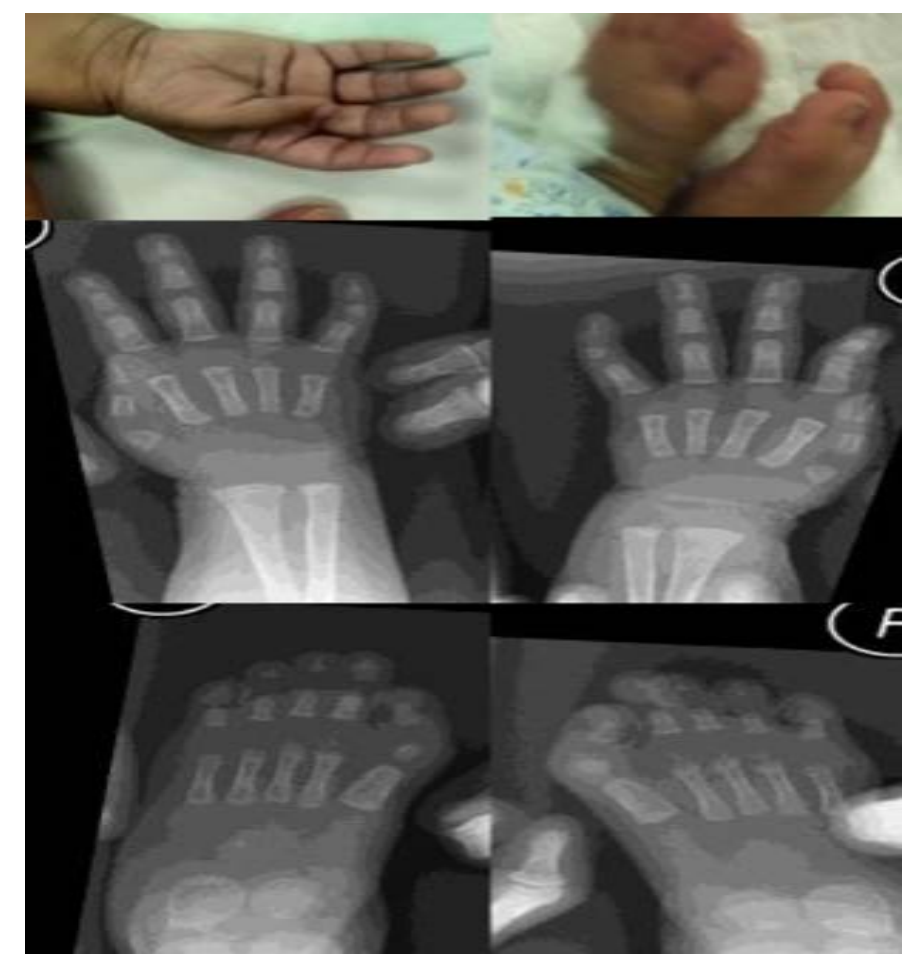

Figure 1. Top: Clinical photograph showing thumbs and great toe in adducted position; Middle: X-ray of hands showing adducted thumbs, clinodactyly; Bottom: X-ray of feet showing hallux abnormality of the great toes. 


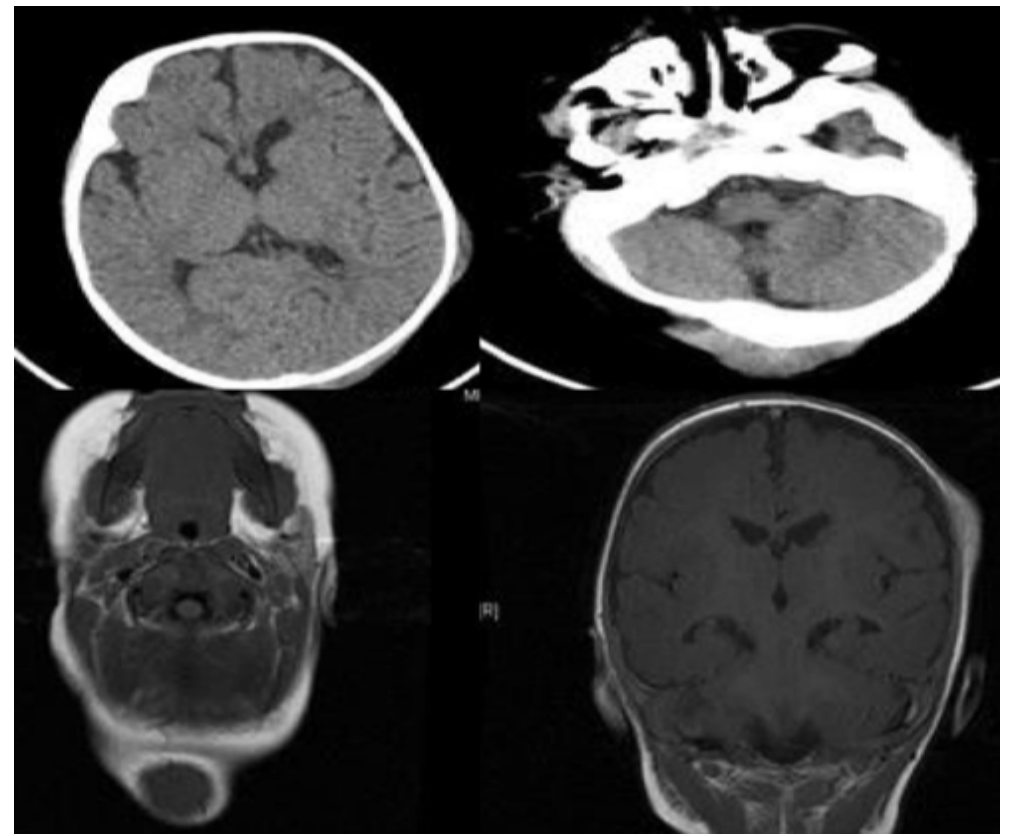

Figure 2. Upper half: CT-scan showing multiple subcutaneous soft tissue masses involving the skull vault at the level of the left Parietal and occipital region. Another relatively larger soft tissue mass is seen in the midline at the level of the posterior fossa; Lower half: MRI head showing a mass within the soft tissues of the left parietal region with intact outer table of the skull vault (coronal view) and another relatively larger one in the midline at the level of craniocervical junction region, with no involvement of the underlying soft tissues or spinal canal.

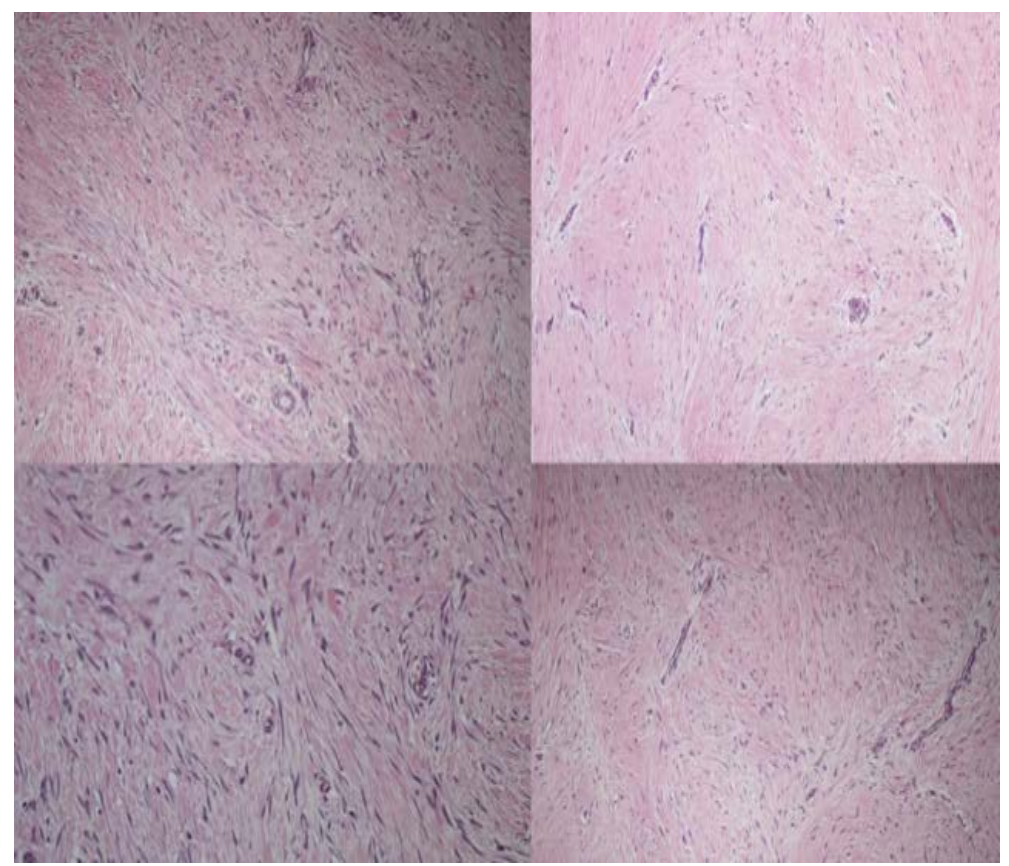

Figure 3. Histopathology of the lesions of nodule consisting of a central area of less differentiated rounder cells with pale cytoplasm and basophilic, small round nuclei, and a peripheral area consisting of spindle cells (myofibroblasts) with eosinophilic cytoplasm and ovoid nuclei arranged in well-demarcated short bundles and fascicles resembling smooth muscle. 


\section{Discussion}

Infantile myofibromatosis is a rare mesenchymal disorder with the growth of one or more benign fibrous tissues of the skin, bone, muscle and viscera [1] [4]. First, described in 1954 in the medical literature by Dr. A. P. Stout, who called it congenital fibromatosis, subsequently several names were used to describe it. However, the term infantile myofibromatosis was coined by Chung and Enzinger in 1981 [5]. Predominantly, it is described in neonates and infants, but there are reports of myofibromatosis occurring in older children and adults. More than $50 \%$ of cases are diagnosed shortly after birth, and up to $90 \%$ are diagnosed before two years of age [5].

It commonly affects the skin, bone, muscle, and soft tissue. In rare cases, the internal organs can be affected (mainly gastrointestinal, pulmonary, and cardiac). The main anatomical sites affected are (in descending order): the head and neck region, the trunk and the limbs. These three regions comprise up to 75\% $80 \%$ of cases [6]. The tumour may occur as solitary or multicentric form, both have same histological features but varied prognosis, besides, visceral involvement is more common with multicentric form. Solitary Infantile myofibromatosis, characterized by a single nodule, is the most frequent presentation, accounting for approximately $75 \%$ of cases [1] [2] [6]. The lesions are firm, fleshy nodules arising from the skin or subcutaneous layer. The superficial lesions can be freely mobile, whereas deeper lesions are immobile. Skin lesions may be crusted or hardened (indurated). The lesions are usually not painful or tender. The overlying skin may be discoloured red or purple and ulcerated. Lesions can also be found in muscle, soft tissue and bone. These tumours do not spread; however, they can grow large enough to cause symptoms by compressing or damaging adjacent organs or different parts of the body [1] [5].

The exact etiology of this disease is not known. Intrauterine estrogen exposure upregulation, leading to proliferation of fibroblast, is suggested as one of the possible etiologies. Most of the cases occur sporadically; however, familial cases have been identified; based on familial occurrences, a genetic cause is also proposed; two different genes, PDGFRB and $\mathrm{NOTCH} 3$, have been determined to cause some of these cases [1] [4] [7]. Histologically, the characteristic feature is the presence of small, round intracytoplasmic inclusions and the presence of uniform, elongated, fusiform, or spindle-shaped fibroblast cells surrounded and separated by abundant collagen. Calcifications are frequent findings, and mitotic activity is slightly increased; nonetheless, it lacks malignant cells, which differentiate fibromatosis from fibrosarcoma [5] [8].

Numerous disorders can resemble the lesions associated with infantile fibromatosis. Such conditions include nodular fascitis, pediatric sarcomas, inflammatory myofibroblastic tumours, desmoids tumours, neurofibromas, hemangiomas, infantile fibrosarcomas, lymphangiomas, fibrous hamartomas of infancy, and epidermoid cysts [1] [4]. A diagnosis of infantile myofibromatosis is based upon the examination of the tissue in a patient with characteristic symp- 
toms and physical examination. Imaging studies are required to visualize the extent of the growth, progression of the growth, and to diagnose the recurrence of the growth. It also is an aid to surgical procedures. A definitive diagnosis of infantile myofibromatosis requires removal and microscopic examination to determine the pathology and distinguishes from the disorders mentioned above, which can mimic like infantile myofibromatosis. Molecular testing detects genetic mutations in specific genes known to cause the disease [1] [7].

Treatment depends upon the location of the lesions. Spontaneous regression can occur in many cases; however, recurrence of the lesions has also been reported [9]. There is no specific treatment available for the affected individuals. In most cases with infantile myofibromatosis, the treatment option is surgery, which is also desired to improve prognosis and prevent complications. Still, lesions may recur in $10 \%$ of cases even after surgery [1] [3] [4]. Some cases may benefit from chemotherapy, in case of visceral involvement, lesions recur after surgical resection or when the lesions are difficult to resect. Children affected have been successfully treated with a combination of drugs like methotrexate and vinblastine. In other cases, interferon alfa, vincristine, actinomycin $\mathrm{D}$, and cyclophosphamide, or their combinations, have also been used successfully. However, these are reserved for individuals with rapid progression of symptoms [10].

The prognosis of Infantile myofibromatosis depends on the type. Generally, solitary and multicentric type without visceral involvement has an excellent outcome, with spontaneous regression of the lesions in one to two years. Whereas, Infantile myofibromatosis with visceral involvement has a poor prognosis and high recurrence risk [4]. Treatment may require the coordinated efforts of a team of specialists. Genetic counselling may be of benefit for families. Psychosocial support for the entire family is essential as well.

Skeletal system involvement, presenting as bone lesions are common with the multicentric type $(20 \%-75 \%)$ but rarely occurs with the solitary type (5\%) of Infantile myofibromatosis [4]. Craniofacial bones are the preferred location for osseous involvement in multicentric type Infantile myofibromatosis; however, many cases have been described with the involvement of long bones of the limbs, small bones of the toes and hands, sporadic cases with involvement of vertebra and iliac bones have also been described in the past. Nevertheless, a detailed search of medical literature, we could not find any case report describing associated skeletal abnormalities with Infantile myofibromatosis. To best of our knowledge, this is the first case-report to report the unique combination of skeletal abnormalities (adducted thumbs, clinodactyly, and bilateral hallux valgus deformity of the great toes) with Infantile myofibromatosis.

\section{Conclusion}

To conclude, we present a rare case of infantile myofibromatosis presenting as multiple lesions in the scalp associated with skeletal abnormalities such as ad- 
ducted thumbs, clinodactyly, and bilateral hallux valgus deformity of the great toes. The unique combination of the distinct skeletal abnormalities in infantile myofibromatosis has not been reported so far. This report emphasizes the possibility of skeletal abnormalities in infantile myofibromatosis.

\section{Declaration of Patient Consent}

The authors certify that they have obtained all appropriate patient consent forms. In the form, the patients gave their consent for their images and other clinical information to be reported in the journal (Signed consent form attached separately).

\section{Contributors' Statement}

Ibrahim Hamama and Tariq Alhazmi conceptualized and designed the report and had full access to the patient data. Ibrahim Hamama and Waseemoddin Patel drafted the manuscript and approved the final version. Tariq Alhazmi reviewed the manuscript and approved the final version.

\section{Conflicts of Interest}

The authors declare no conflicts of interest regarding the publication of this paper.

\section{References}

[1] Mashiah, J., Hadj-Rabia, S., Dompmartin, A., et al. (2014) Infantile Myofibromatosis: A Series of 28 Cases. Journal of the American Academy of Dermatology, 71, 264-270. https://doi.org/10.1016/j.jaad.2014.03.035

[2] Larralde, M., Hoffner, M.V., Boggio, P., et al. (2010) Infantile Myofibromatosis: Report of Nine Patients. Pediatric Dermatology, 27, 29-33. https://doi.org/10.1111/j.1525-1470.2009.01073.x

[3] Maby, A., Guay, B. and Thuot, F. (2019) Infantile Myofibromatosis Treated by Mandibulectomy and Staged Reconstruction with Submental Flap and Free Fibula Flap: A Case Report. Journal of Otolaryngology Head Neck Surgery, 48, Article No. 14. https://doi.org/10.1186/s40463-019-0333-Z

[4] Wu, W., Chen, J., Cao, X., et al. (2013) Solitary Infantile Myofibromatosis in the Bones of the Upper Extremities: Two Rare Cases and a Review of the Literature. Oncology Letters, 6, 1406-1408. https://doi.org/10.3892/ol.2013.1584

[5] Al-Salem, A.H. (2014) Infantile Fibromatosis and Myofibromatosis. In: Al-Salem, A.H., Ed., An Illustrated Guide to Pediatric Surgery. Springer International Publishing, Cham, 57-61. https://doi.org/10.1007/978-3-319-06665-3_9

[6] Fau, C.E. and Enzinger, F.M. (1981) Infantile Myofibromatosis. Cancer, 48, 1807-1818.

https://doi.org/10.1002/1097-0142(19811015)48:8<1807::AID-CNCR2820480818>3. $\underline{0 . \mathrm{CO} ; 2-\mathrm{G}}$

[7] Martignetti, J.A., Tian, L., Li, D., et al. (2013) Mutations in PDGFRB Cause Autosomal-Dominant Infantile Myofibromatosis. American Journal of Human Genetics, 92, 1001-1007. https://doi.org/10.1016/j.ajhg.2013.04.024

[8] Hausbrandt, P.A., Leithner, A., Beham, A., et al. (2010) A Rare Case of Infantile 
Myofibromatosis and Review of Literature. Journal of Pediatric Orthopaedics B, 19, 122-126. https://doi.org/10.1097/BPB.0b013e32832e4756

[9] Kikuchi, K., Abe, R., Shinkuma, S., et al. (2011) Spontaneous Remission of Solitary-Type Infantile Myofibromatosis. Case Reports in Dermatology, 3, 181-185. https://doi.org/10.1159/000331325

[10] Wu, S.Y., McCavit, T.L., Cederberg, K., Galindo, R.L. and Leavey, P.J. (2015) Chemotherapy for Generalized Infantile Myofibromatosis with Visceral Involvement. Journal of Pediatric Hematology/ Oncology, 37, 402-405.

https://doi.org/10.1097/MPH.0000000000000132

\section{Abbreviations}

AP View: Antero-Posterior View

CT Scan: Computerized Tomography Scan

MRI: Magnetic Resonance Imaging 